\title{
Coevolutionary modeling in network formation
}

\author{
Ibrahim Al-Shyoukh*, Georgios Chasparis ${ }^{\dagger}$, and Jeff S. Shamma*‡ \\ * School of Electrical and Computer Engineering, Georgia Institute of Technology, Atlanta, GA 30332 \\ $\dagger$ Department of Data Analysis Systems, Software Competence Center Hagenberg GmbH, Hagenberg, Austria \\ ${ }^{\ddagger}$ CEMSE Division, King Abdullah University of Science and Technology (KAUST), Thuwal, Saudi Arabia
}

\begin{abstract}
Network coevolution, the process of network topology evolution in feedback with dynamical processes over the network nodes, is a common feature of many engineered and natural networks. In such settings, the change in network topology occurs at a comparable time scale to nodal dynamics. Coevolutionary modeling offers the possibility to better understand how and why network structures emerge. For example, social networks can exhibit a variety of structures, ranging from almost uniform to scale-free degree distributions. While current models of network formation can reproduce these structures, coevolutionary modeling can offer a better understanding of the underlying dynamics. This paper presents an overview of recent work on coevolutionary models of network formation, with an emphasis on the following three settings: (i) dynamic flow of benefits and costs, (ii) transient link establishment costs, and (iii) latent preferential attachment.
\end{abstract}

\section{INTRODUCTION}

Dynamic models for modeling network formation, where nodes/agents have the power to form or severe links with other nodes/agents over time, have served as a natural tool for capturing the establishment of real-world social networks. Questions such as how certain network structures form and how these structures may emerge through certain agent-level decision processes arise naturally. Recent efforts in modeling such endogenous processes in network formation emphasize the dynamic nature of the formation process, considering dynamic/evolutionary models. In such models, agents react adaptively to the current form of the network by forming new links or severing some of the existing ones. Examples of such models include [1], [2], [3], [4], [5], [6], [7], [8], [9], [10], [11], [12], [13], [14], emphasizing the role of agent-level decisions on the observed structures.

In such settings, agents make unilateral decisions about their links based on their own preferences governed by utility functions that depend on the network topology. For example, preferential attachment [15] uses degree distributions, whereas a strategic connections model [16] uses internode distances. An implicit assumption here is that the utility derived from a network topology is an instantaneous function of the network topology. Stated differently, if the utility is a dynamic function of the network topology, these dynamics evolve much faster than changes in network topology, resulting in a time-scale separation between agents revising their decisions and the utility dynamic process. In the absence of time-scale separation, this combination of evolving network and dynamic utility dependence necessitates a coevolutionary analysis.

A general description of coevolutionary systems is that there is a dynamic process reacting to network topology and a network topology reacting to the states of these dynamics (cf., [17]). In the above discussion of network formation, the dynamics process was the evolving utilities, and the network topology evolves in reaction to these utilities. Another setting where coevolution arises is in diffusion models of consensus, gossip, averaging, etc. (e.g., [18], [19], [20]). In this setting, there is an underlying graph over which agents (nodes) evolve a local state by averaging over their neighbors in the graph. In much of this work, the network topology is fixed. In a coevolutionary setting, the topology evolves in reaction to the evolving states. One such example is a graph based on proximity as in the Hegselmann-Krause dynamics [21]. Here, there is an edge between two agents only if their states are close. Accordingly, the states evolve according to the topology and the topology evolves according to the states, and the ensuing analysis is significantly more complicated [22], [23], [24]. One can interpret a fixed topology as a slowly varying topology as compared to the averaging state dynamics, but again, the absence of a time-scale separation necessitates a coevolutionary analysis.

This paper presents a summary of selected recent work that addresses such coevoltuionary behavior in network formation. We consider three settings. In the first setting [25], the benefits and costs of links evolve as a dynamic function of the network topology. Furthermore, agents are impatient to wait for these values to settle. In the second setting [26], we consider a setup where benefits and maintenance costs are an instantaneous function of network topology, but introduce a link establishment cost that reflects an agent's familiarity with other agents. Finally, we consider a coevolutionary form of preferential attachment in which incoming nodes are unable to observe the actual degree of a node, but rather a latent version of the degree.

\section{Dynamic Flow of Benefits And Coevolution}

In networks involving the exchange of benefits or goods, the benefits may not be transferred immediately, i.e., the benefits between two nodes will flow over time. Traditional strategic network formation models of such systems do not account for the dynamics of flow of benefits and assume the immediate transfer of benefits. Here, we present an overview of our recent results in [25] in which we present a model that captures the emergent behaviors of network coevolution under dynamic benefit flows.

Consider a generic form of utility function that captures the 
balance between benefits and costs is

$$
u_{i}\left(g_{i}, g_{-i}\right)=R_{i}(g)-C_{i}(g)
$$

where $u_{i}(\cdot)$ is the utility of the $i^{\text {th }}$ agent, $g_{i}$ is the chosen neighbor set of the $i^{\text {th }}$ agent, and $g_{-i}$ are the chosen neighbor sets of other agents. The combined set of choices/actions, $g_{i}$, of all agents $i \in\{1,2, \ldots, N\}$, where $N$ is the number of nodes, defines a network/graph $g$ which we identify as $g=\left(g_{i}, g_{-i}\right)$. Accordingly, $R_{i}(g)$ and $C_{i}(g)$ are the benefits and costs, respectively, to the $i^{\text {th }}$ agent as a function of the network, $g$. This formulation has been studied extensively in the network formation literature [27], [1], [6], [6], [3], [2], [4], [26]. As previously discussed, the link formation dynamics implicitly presume that such benefits and costs are constant functions of the network topology that arise instantaneously upon link formation. Such restriction permits establishing a connection between static equilibrium notions, such as the Nash equilibrium of the associated game, with the convergence properties of the considered dynamics, such as the bestresponse dynamics of [1]. On the other hand, such restriction ignores potential temporal variations of the utility function itself and their impact on the observed asymptotics.

For a given network $g=\left(g_{i}, g_{-i}\right)$, and given the benefits $b_{i}$, the utility for node $i$ can be given by

$$
u_{i}\left(b_{i}, g_{i}\right)=\sum_{j} b_{i j}-c \mu\left(g_{i}\right) .
$$

In the instantaneous benefit flow model, the feedback law to select strategies assumed an instantaneous realization of the full benefits from other nodes. Alternatively, the strategy of a node can dynamically depend on the available benefits at a given time. Here, a node can be selecting a strategy at a given point in time such that it maximizes a utility dependent cost function.

In particular, consider the following benefit flow dynamics

$$
b_{i j}^{t}=f\left(b_{i j}^{t-1}, g_{i}, g_{-i}\right)= \begin{cases}\phi_{1}, & \delta^{d_{i j}} \geq b_{i j}^{t-1} \\ \phi_{2}, & \delta^{d_{i j}}<b_{i j}^{t-1}\end{cases}
$$

such that $\beta_{i}, \alpha_{i} \in[0,1], \alpha_{1} \leq \alpha_{2} \leq \ldots$ and $\beta_{1} \geq \beta_{2} \geq \ldots$, where $\phi_{1}=\alpha_{d_{i j}} b_{i j}^{t-1}+\left(1-\alpha_{d_{i j}}\right) \delta^{d_{i j}}, \phi_{2}=\beta_{d_{i j}} b_{i j}^{t-1}+(1-$ $\left.\beta_{d_{i j}}\right) \delta^{d_{i j}}, d_{i j}$ is the shortest distance between nodes $i$ and $j$.

Upon establishing a link, the benefits will flow from node $j$ to node $i$ and will be fully realized asymptotically. Alternatively, when a path between two nodes is severed, then the benefits available from a node to another are not lost immediately, but are forgotten over time. Here, the rate is also distance dependent.

For myopic decision makers that seek to maximize the projected utility in the next time step assuming the strategies of the other nodes remain fixed, we can define the utility for a given strategy $g_{i}$ and benefits vector $b_{i}$ at time $t-1$ to be

$$
u_{i}\left(g_{i}, g_{-i}^{t-1}, b_{i}^{t-1}\right)=\sum_{j} f\left(b_{i j}^{t-1}, g_{i}, g_{-i}^{t-1}\right)-c \mu\left(g_{i}\right) .
$$

Let $\mathcal{G}_{i}$ denote the set of all possible pure strategies of node.
A randomly selected node, at time steps $t=1,2, \ldots$, plays a best response to the currently observed benefit flow and network topology,

$$
g_{i}^{t}=\operatorname{BR}\left(g_{-i}^{t-1}, b_{i}^{t-1}\right) \in \arg \max _{g_{i} \in \mathcal{G}_{i}} u_{i}\left(g_{i}, g_{-i}^{t-1}, b_{i}^{t-1}\right) .
$$

Definition 2.1: The pair $\left(B^{*}, g^{*}\right)$ is an equilibrium of the coupled dynamics in (1) and (3) if $\forall i \in \mathcal{N}, g_{i}^{*}=\operatorname{BR}\left(g_{-i}^{*}, b_{i}^{*}\right)$ and $\forall j b_{i j}^{*}=f\left(b_{i j}^{*}, g_{i}^{*}, g_{-i}^{*}\right)$. The equilibrium is strict if and only if $u\left(g_{i}^{*}, g_{-i}^{*}, b_{i}^{*}\right)-u\left(g_{i}^{\prime}, g_{-i}^{*}, b_{i}^{*}\right)>0, \forall g_{i}^{\prime} \in \mathcal{G}_{i} \backslash g_{i}^{*}, \forall i \in$ $\mathcal{N}$

Here the equilibrium involves both network topology $g^{*}$ and a steady-state benefit flow $B^{*}$. One class of equilibria that can emerge is when the topology of the network remains unchanged, i.e., $g^{t}=g^{*}, \forall t \geq t_{0}$ for some network topology $g^{*}$. As a consequence, the shortest distances between nodes remain unchanged, i.e, $d_{i j}\left(g_{i}^{t}, g_{-i}^{t}\right)=d_{i j}\left(g_{i}^{*}, g_{-i}^{*}\right), \forall i, j$ and $\forall t \geq t_{0}$. Therefore, the benefits for each node will correspond to $b_{i j}^{*}=\delta^{d_{i j}\left(g_{i}^{*}, g_{-i}^{*}\right)}$.

One of the questions to consider is whether the coevolutionary dynamics can induce some network topologies to become equilibria while they are not equilibria of the noncoevolutionary network formation (static game). In the following we show through an example that given a common set of parameters, such topologies exist.

Proposition 2.1: For $N=3$, if $\left(1-\beta_{\infty}\right) \delta \geq c \geq(1-$ $\left.\alpha_{1}\right)\left(\delta-\delta^{2}\right)$, and $\beta_{2}<\alpha_{1}$, then the pair $\left(B^{*}, g^{*}\right)$ given by

$$
g^{*}=((1,0),(1,1),(0,1)), \quad B_{g}^{*}=\left[\begin{array}{ccc}
1 & \delta & \delta^{2} \\
\delta & 1 & \delta \\
\delta^{2} & \delta & 1
\end{array}\right],
$$

is an equilibrium of the coupled dynamics in (1) and (3), and $g^{*}$ is not a Nash equilibrium of the static game when $\alpha_{1}>0$.

Another important question to consider is whether such equilibria are stable in that a given network topology with benefit flows close enough to the equilibrium values will remain unchanged.

Proposition 2.2: Let $\left(g^{*}, B^{*}\right)$, where $g^{*}=\left(g_{i}^{*}, g_{-i}^{*}\right), B^{*}=$ $\left[b_{i j}^{*}\right]$, be a strict equilibrium such that $\forall i, u_{i}\left(g_{i}^{*}, g_{-i}^{*}, b_{i}^{*}\right)-$ $u_{i}\left(g_{i}, g_{-i}^{*}, b_{i}^{*}\right) \geq \gamma, \forall g_{i} \in \mathcal{G}_{i} \backslash g_{i}^{*}$, for some $\gamma>0$. Also, let $g_{t_{0}}=g^{*}$ and $b_{i j}^{t_{0}}=b_{i j}^{*} \pm \epsilon \forall i, j$, for some time $t_{0}$. If $\epsilon$ is sufficiently small, then $g_{t}=g^{*}, \forall t \geq t_{0}$, and $\lim _{t \rightarrow \infty} b_{i j}^{t}=$ $b_{i j}^{*}, \forall i, j$.

\section{ESTABLISHMENT COST, EXPLORATION BENEFIT AND STABLE NETWORKS}

Most models of network formation assume some cost associated with link formation. Typically, these are maintenance costs, which reflect the cost of having a link. Another important type of cost is establishment costs, i.e., the cost to form a new link. Unlike maintenance costs, establishment costs are transient phenomena in that once a link is established, the impact of the establishment cost diminishes over time. We present recent work that models establishment cost in terms of familiarity. Nodes have associated familiarity with other nodes based on a fading memory of past links. Establishing a new link has a cost that is greater for nodes that are less 
familiar. Once the link is established, familiarity increases, and so the establishment cost decays. Alternatively, there can be an establishment reward, in which nodes are encouraged to explore by connecting to unfamiliar nodes. We analyze and illustrate establishment costs on a dynamic network formation process and contrast the behaviors with and without establishment costs.

To capture such dynamic aspects of the utility function, we define state-based utility functions [28] as follows.

Definition 3.1 (State-based utility function): A state-based utility function maps $v_{i}: \mathcal{A} \times \mathcal{X}_{i} \rightarrow \mathbb{R}_{+}$with $v_{i}\left(\alpha, x_{i}\right)$ being the payoff of agent $i$ at joint action $\alpha$ and at state $x_{i}$.

The definition of the state vector is open-ended. For capturing a familiarity dependence of the cost function, we may define the state vector as a probability distribution over the available link selections $\mathcal{A}_{i}$, i.e., $x_{i} \in \Delta\left(\left|\mathcal{A}_{i}\right|\right)$, where $\Delta(n)$ denotes the probability simplex of dimension $n$. In that case, the familiarity vector $x_{i}$ corresponds to a unit vector, say $e_{j}$, i.e., the "familiarity" weighting of agent $i$ with action $j$ is 1 , while the corresponding weighting with other actions is 0 . By abusing notation, we will also write $x_{i}=\alpha_{i}$ to denote familiarity weighting 1 with the action selection $\alpha_{i}$.

Stability notions, such as the Nash equilibrium may be extended to capture the state dependency of the utility function. In the case of the familiarity dependence of the cost function, we may define a better reply as follows:

Definition 3.2 (State-based better reply): The better reply set of agent $i \in \mathcal{I}$ to an action profile $\alpha=\left(\alpha_{i}, \alpha_{-i}\right) \in \mathcal{A}$ is a function $\mathrm{BR}_{i}: \mathcal{A} \rightarrow \mathcal{A}_{i}$ such that for any $\alpha_{i}^{*} \in \mathrm{BR}_{i}(\alpha)$ we have

$$
v_{i}\left(\left(\alpha_{i}^{*}, \alpha_{-i}\right), \alpha_{i}\right)>v_{i}\left(\left(\alpha_{i}, \alpha_{-i}\right), \alpha_{i}\right) .
$$

Note that better reply is a set-valued function and might be empty. Furthermore, when we evaluate the better reply set of an agent $i$ to an action profile $\alpha=\left(\alpha_{i}, \alpha_{-i}\right)$, the underlying familiarity state in the agent's state-based utility is assumed to be the corresponding action of that agent, $\alpha_{i}$.

Based on this definition of better reply, we introduce the notion of a "stable" action profile by extending the definition of a Nash equilibrium to state-based utility functions.

Definition 3.3 (State-based Nash equilibrium): An action profile $\alpha^{*}$ is a (state-based) Nash equilibrium if $\operatorname{BR}_{i}\left(\alpha^{*}\right)=\varnothing$ for every $i \in \mathcal{I}$, i.e.,

$$
v_{i}\left(\left(\alpha_{i}^{*}, \alpha_{-i}^{*}\right), \alpha_{i}^{*}\right) \geq v_{i}\left(\left(\alpha_{i}^{\prime}, \alpha_{-i}^{*}\right), \alpha_{i}^{*}\right),
$$

for all $\alpha_{i}^{\prime} \in \mathcal{A}_{i} \backslash\left\{\alpha_{i}^{*}\right\}$ and $i \in \mathcal{I}$. Likewise, a strict (statebased) Nash equilibrium satisfies the strict inequality in (5).

Implicitly here we assume that equilibrium may only be established at a state of maximum familiarity, while deviations from it will result to connecting with unfamiliar nodes.

Example: The state-based utility function of agent $i$ is a function of the form $v_{i}: \mathcal{A} \times \Delta\left(\left|\mathcal{A}_{i}\right|\right) \rightarrow \mathbb{R}_{+}$, where $v_{i}\left(\alpha, x_{i}\right) \triangleq R_{i}(\alpha)-C_{i}\left(\alpha_{i}, x_{i}\right)$. Links will be considered unidirectional, where the direction of a link determines the direction of benefits flow. In particular, the function $R_{i}$ : $\mathcal{A} \rightarrow \mathbb{R}_{+}$is the reward of agent $i$ and will be defined here as the number of nodes accessed from node $i$ through its direct/indirect connections [27]. Informally, a link with another agent inherits the benefits available to that agent via its own links.

The cost function of agent $i, C_{i}: \mathcal{A}_{i} \times \Delta\left(\left|\mathcal{A}_{i}\right|\right) \rightarrow \mathbb{R}_{+}$will capture establishment cost based on link familiarity and may be defined as follows:

$$
C_{i}\left(\alpha_{i}, x_{i}\right) \triangleq \kappa_{0}\left|\alpha_{i}\right|+\kappa_{1} \psi_{i}\left(\alpha_{i}\right)^{\mathrm{T}}\left(\mathbf{1}-\psi_{i}\left(x_{i}\right)\right),
$$

with $\kappa_{0} \geq 0, \kappa_{1} \in \mathbb{R}$, and $\psi_{i}: \Delta\left(\left|\mathcal{A}_{i}\right|\right) \rightarrow \mathbb{R}^{\left|\mathcal{N}_{i}\right|}$ defined by $\left[\psi_{i}\left(x_{i}\right)\right]_{j} \triangleq \sum_{\left\{a \in \mathcal{A}_{i}: j \in a\right\}} x_{i a}$, where we are using $a$ as both an index, as in $x_{i a}$, and a set, as in $j \in a \in 2^{\mathcal{N}_{i}}$. In words, $\left[\psi_{i}\left(x_{i}\right)\right]_{j}$ denotes the probability that agent $i$ will form a link to neighbor $j$ based on the distribution $x_{i}$. The term $(1-$ $\left.\psi_{i}\left(x_{i}\right)\right)^{\mathrm{T}} \psi_{i}\left(\alpha_{i}\right)$ grows with misalignment of the action $\alpha_{i}$ with the distribution $x_{i}$. In the perfectly aligned case, for any $\alpha_{i} \in$ $\mathcal{A}_{i}$ (viewed as a vertex of $\left.\Delta\left(\left|\mathcal{A}_{i}\right|\right)\right), \psi_{i}\left(\alpha_{i}\right)^{\mathrm{T}}\left(\mathbf{1}-\psi_{i}\left(\alpha_{i}\right)\right)=0$ whereas in the worst case, $\max _{x_{i}} \psi_{i}\left(\alpha_{i}\right)^{\mathrm{T}}\left(\mathbf{1}-\psi_{i}\left(x_{i}\right)\right)=\left|\alpha_{i}\right|$.

The first part of the cost function (6) corresponds to the cost of maintaining the currently established links. The state $x_{i}$ reflects familiarity with a particular set of links. Accordingly, the second part corresponds to an establishment cost. The establishment cost models possible inertia of the system. When $\kappa_{1}>0$, this term represents the effort necessary to establish a new link, whereas in the case $\kappa_{1}<0$, it represents incentives to explore.

The question that naturally emerges is whether the familiarity state may alter the stability properties of networks that are stable when the familiarity term is non present $\left(\kappa_{1}=0\right)$, i.e., they are Nash equilibria under no state dependence. We start with considering positive establishment cost.

Proposition 3.1 (Nash networks for small $\kappa_{1}>0$ ): For $\kappa_{0}+\kappa_{1}<1$, there exists $\bar{\kappa}_{1}>0$ such that:

1) If $\alpha$ is not a Nash network for $\kappa_{1}=0$, then $\alpha$ is not a Nash network for $\kappa_{1} \in\left(0, \bar{\kappa}_{1}\right)$;

2) If $\alpha$ is a Nash network for $\kappa_{1}=0$, then $\alpha$ is a strict Nash network for $\kappa_{1} \in\left(0, \bar{\kappa}_{1}\right)$.

In other words, establishment cost may strengthen stability of Nash networks with no familiarity dependence. On the other hand, the case of negative establishment cost, i.e., $\kappa_{1}<0$, can be viewed as rewarding exploration. The consequences are as follows.

Proposition 3.2 (Nash networks for small $\kappa_{1}<0$ ): For $\kappa_{0}+\kappa_{1}<1$, there exists a $\underline{\kappa}_{1}<0$ such that:

1) If $\alpha$ is not a Nash network for $\kappa_{1}=0$, then $\alpha$ is not a Nash network for $\kappa_{1} \in\left(\underline{\kappa}_{1}, 0\right)$;

2) If $\alpha$ is a non-strict Nash network for $\kappa_{1}=0$, then $\alpha$ is not a Nash network for $\kappa_{1} \in\left(\underline{\kappa}_{1}, 0\right)$;

3) If $\alpha$ is a strict Nash network for $\kappa_{1}=0$, then $\alpha$ is a strict Nash network $\kappa_{1} \in\left(\underline{\kappa}_{1}, 0\right)$.

The opposite phenomenon is observed here, since rewarding exploration may result in weakening stability of Nash networks with no familiarity dependence.

In either one of the above cases, we observe how a statedependent cost function based on familiarity may alter the 
stability properties of networks, emphasizing the significant role of transition phenomena on the emerging behavior.

\section{Coevolutionary Preferential Attachment}

Another fundamental model of network formation is that of preferential attachment [15]. Here a new node connects to existing nodes within the network based on the degree of each node. In many real-life networks, the actual degree of a given node is not available; rather a proxy of the degree can be measured or estimated. This perceived degree can lag the actual degree. For example, in citation networks, knowledge of the number of citations a given paper has can be inaccurate. The estimate of the node degree can be less than the actual number due to delays in propagating and accumulating citation info. Despite this uncertainty, citation networks exhibit a degree distribution similar to that of standard preferential attachment. We introduce a new dynamic preferential attachment model to capture the lack of knowledge of node degree and the dynamic nature of update of estimates of node degrees. The resulting system exhibits a power-law distribution of node degrees.

In the Barabasi-Albert model of network formation, starting with a small number of nodes $\left(m_{0}\right)$, a new node arrives at every time step and forms $m \leq m_{0}$ links (edges) with existing nodes, the probability of forming a link with an existing nodes is proportional to the state of the node here taken as the degree, giving rise to preferential attachment. Hence, a node with a higher degree has a higher probability of getting a new link to it. For node $i$ with degree $k_{i}$, the probability that an incoming node forms a link with node $i$ is $\Pi\left(k_{i}\right)=k_{i} / \sum_{j} k_{j}$.

Consider the case when the state of a given node is the apparent attractivity of a node, and is a delayed response of the degree of the node. That is, an incoming node cannot see the exact degree or attractivity of a given node, rather, it sees a delayed response of the degree of the node. Let $c_{i}$ be the apparent attractivity of a given node such that

$$
c_{i}(t+1)=\alpha c_{i}(t)+(1-\alpha) k_{i}(t),
$$

where $\alpha \in[0,1]$, with $\alpha=0$ corresponding to the original Barabasi-Albert model.

The coevolutionary dynamics of state dependent network formation and network dependent state evolution presents an interesting problem that requires further analysis, especially when the time-scales of the two equations are comparable and there is no separation of time-scales. If the attractivity of a node is updated upon the arrival of any new node to the system, then the attractivity will be updated fast enough for most nodes and a separation of time scales arises. However, whenever the attractivity is updated only upon an incoming link to the node itself, then the times scales of the two processes will be comparable, resulting in coevolutionary dynamics.

The derivation of a closed form representation of the limiting distribution of the coevolutionary model is a challenge and remains open. We conducted numerical experiments to to examine the emergent behavior. The simulation results show that the coevolutionary model exhibits a degree distribution that deviates from that of standard preferential attachment.
While the slope for higher values of $k(k \geq 12)$ is comparable to standard preferential attachment, the distribution exhibit a different behavior for low values of $k$. There is also an increase in the number of super hubs (nodes with very high degree $k$ ). A similar increase in super hubs was observed previously due to multi scaling and competitiveness [29].

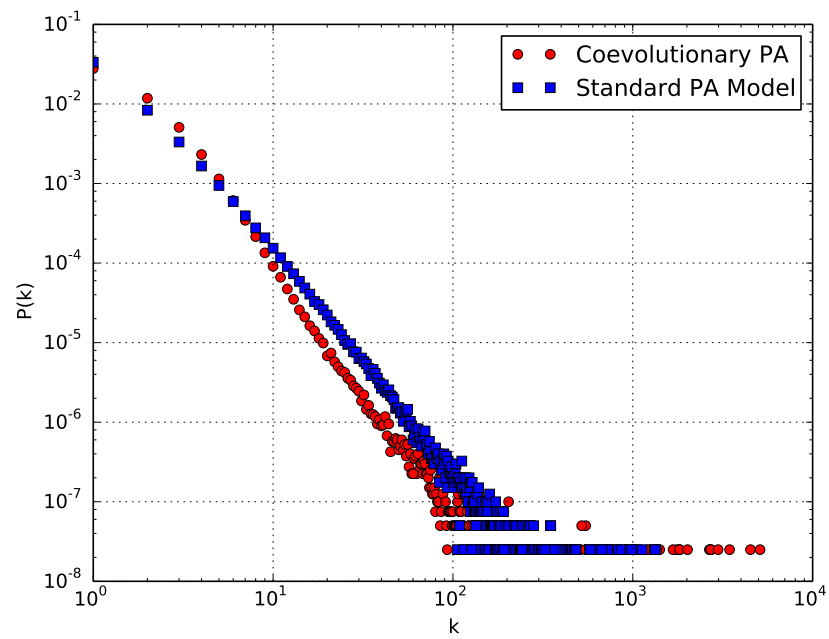

Fig. 1. Degree distribution for the coevolutionary preferential attachment model compared to standard preferential attachment when $\alpha=0.9$.

\section{CONCluding Remarks}

We have reviewed three settings of coevolutionary dynamics in network formation: (i) dynamic flow of benefits and costs, (ii) transient link establishment costs, and (iii) latent preferential attachment. Clearly, much work remains both in the analysis of such models and their relation to available network data. Coevolutionary modeling offers the possibility to better understand how and why network structures emerge. For example, social networks can exhibit a variety of structures, e.g., ranging from almost uniform to scale-free degree distributions [30]. While current models of network formation can reproduce these structures, coevolutionary modeling can offer a better understanding of the underlying dynamics. Likewise, coevolutionary modeling can provide insights into other phenomena such as cascading failure in networks. The model of [31] compares the robustness of different network structures, in which nodes are randomly and exogenously assigned immunity levels. This exogenous assignment allows for the questionable circumstance of a node with high centrality to have low immunity. Alternatively, coevolutionary modeling could offer insights into the interplay between centrality and immunity.

Index Terms-Network science, Network formation, Evolutionary game theory

\section{ACKNOWLEDGEMENT}

Research supported in part by AFOSR/MURI \#FA9550-091-0538 and \#FA9550-10-1-0573. 


\section{REFERENCES}

[1] V. Bala and S. Goyal, "A noncooperative model of network formation," Econometrica, vol. 68, no. 5, pp. 1181-1229, 2000.

[2] B. Skyrms and R. Pemantle, "A dynamic model of social network formation," Proc. of the National Academy of Sciences of the USA, vol. 97, pp. 9340-9346, 2000.

[3] M. Jackson and A. Watts, "The evolution of social and economic networks," Journal of Economic Theory, vol. 106, no. 2, pp. 265-295, 2002.

[4] P. Bonacich and T. Liggett, "Asymptotics of a matrix-valued markov chain arising in sociology," Stochastic Processes and Their Applications, vol. 104, pp. 155-171, 2003.

[5] E. Arcaute, R. Johari, and S. Mannor, "Network formation: Bilateral contracting and myopic dynamics," Automatic Control, IEEE Transactions on, vol. 54, no. 8, pp. 1765-1778, 2009.

[6] N. Olaizola and F. Valenciano, "One-way flow network formation under constraints," University of the Basque Country, Working Paper IL. 58/12, 2012.

[7] M. O. Jackson, "A survey of network formation models: stability and efficiency," Group formation in economics: networks, clubs and coalitions. Cambridge University Press, Cambridge, pp. 11-57, 2005.

[8] E. Tardos and T. Wexler, "Network formation games and the potential function method," Algorithmic Game Theory, pp. 487-516, 2007.

[9] F. Bloch and M. O. Jackson, "The formation of networks with transfers among players," Journal of Economic Theory, vol. 133, no. 1, pp. 83110, 2007.

[10] M. O. Jackson, Social and economic networks. Princeton University Press, 2010.

[11] N. A. Christakis, J. H. Fowler, G. W. Imbens, and K. Kalyanaraman, "An empirical model for strategic network formation," National Bureau of Economic Research, Tech. Rep., 2010.

[12] R. Cowan, "Network models of innovation and knowledge diffusion," Clusters, networks and innovation, pp. 29-53, 2005.

[13] H. P. Young, "The dynamics of social innovation," Proceedings of the National Academy of Sciences, vol. 108, no. Supplement 4, pp. 21285 $21291,2011$.

[14] _ Individual strategy and social structure: An evolutionary theory of institutions. Princeton University Press, 2001.

[15] A. Barabási and R. Albert, "Emergence of scaling in random networks," Science, vol. 286, pp. 509-512, 1999.

[16] V. Bala and S. Goyal, "A non-cooperative model of network formation," Econometrica, vol. 68, pp. 1181-1229, 2000.

[17] T. Gross and B. Blasius, "Adaptive coevolutionary networks: a review," Journal of The Royal Society Interface, vol. 5, no. 20, pp. 259-271, 2008.

[18] V. Blondel, J. Hendrickx, A. Olshevsky, and J. Tsitsiklis, "Convergence in multi agent coordination, consensus, and flocking," in Proceedings of the 44th IEEE Conference on Decision and Control, vol. 44, no. 3, Seville, Spain, 2005, pp. 2996-3000.

[19] A. Jadbabaie, J. Lin, and S. A. Morse, "Coordination of groups of mobile agents using nearest neighbor rules," IEEE Transactions on Automatic Control, vol. 48, no. 6, pp. 988-1001, Jun. 2003.

[20] R. Olfati-Saber, J. A. Fax, and R. M. Murray, "Consensus and cooperation in networked multi-agent systems," Proceedings of the IEEE, vol. 95, no. 1, pp. 215-233, 2007.

[21] R. Hegselmann and U. Krause, "Opinion dynamics and bounded confidence: Modes, analysis, and simulation," Journal of Artificial Societies and Social Simulation, vol. 5, no. 3, 2002.

[22] A. Mirtabatabaei and F. Bullo, "Opinion dynamics in heterogeneous networks: Convergence conjectures and theorems," SIAM Journal on Control and Optimization, vol. 50, no. 5, pp. 2763-2785, 2012.

[23] V. Blondel, J. Hendrickx, and J. Tsitsiklis, "On Krause's consensus formulation model with state-dependent connectivity," IEEE Transactions on Automatic Control, vol. 54, pp. 2586-2597, 2009.

[24] B. Touri and C. Langbort, "On indigenous random consensus and averaging dynamics," in Proceedings of the 52nd IEEE Conference on Decision and Control, Florence, Italy, 2013, pp. 6208-6212.

[25] I. Al-Shyoukh and J. Shamma, "A coevolutionary model of strategic network formation," in Complex Networks V, P. C. et al., Ed. Springer, 2014.

[26] G. Chasparis and J. Shamma, "Network formation: Neighborhood structures, establishment costs, and distributed learning," IEEE Transactions in Systems, Man and Cybernetics - Part B: Cybernetics, vol. 43, no. 6 , pp. 1950-1962, 2013

[27] M. Jackson and A. Wolinsky, "A strategic model of social and economic networks," Journal of Economic Theory, vol. 71, pp. 44-74, 1996.

[28] J. Marden, "State based potential games," Automatica, vol. 48, pp. 30753088, 2012.

[29] G. Bianconi and A.-L. Barabasi, "Competition and multiscaling in evolving networks," Europhysics Letters, vol. 54, no. 4, pp. 436-442, 2001.

[30] M. Jackson and B. Rogers, "Meeting strangers and friends of friends: How random are social networks," American Economic Review, vol. 3, pp. 890-915, 2007.

[31] L. Blume, D. Easley, J. Kleinberg, R. Kleinberg, and E. Tardos, "Which networks are least susceptible to cascading failures?" in Proceedings of the 52nd Annual Symposium on Foundations of Computer Science (FOCS2011), October 2011, pp. 393-402. 